\title{
Guiding authors to Open Access
}

\author{
Guided Open Access is a new publishing option offered at Nature Genetics. Authors can submit once and be \\ simultaneously considered by three journals. Editorial collaboration and a single submission system combine to \\ make the publication process easier and faster.
}

$\mathrm{N}$ ature Genetics now offers three publishing options for new manuscripts submitted in 2021: the traditional (subscription) model, Open Access and Guided Open Access. Whereas the first two options are well-known parts of the publishing landscape, the Guided Open Access option is different.

In Guided Open Access, a manuscript is submitted once and is evaluated by editors from three different journals that publish in the appropriate subject area. Genetics manuscripts submitted to Nature Genetics will be assessed by editors from Nature Genetics, Nature Communications and Communications Biology. Editors will work collaboratively to help authors find the best home for their manuscript. All manuscripts that are within scope and determined to be suitable for at least one of the journals will be sent for review. A single handling editor from one of the journals will champion the manuscript and oversee the peer-review process, but decisions will be made through discussion with editors at all three journals. Authors will receive a detailed 'Editorial Assessment Report' containing reviewer comments annotated by the editors, tailored guidance recommending the next steps and a notification of which journal(s) would like to invite a revision addressing the reviewer comments. Articles accepted at Nature Genetics will be published Open Access with an Article Processing Charge (APC) approximately $50 \%$ less than that for the standard Open Access option. Thus, Guided Open Access offers the convenience of a single submission, personalized guidance from editors at different journals and the opportunity to publish Open Access in Nature Genetics at a reduced APC.

The cost for Guided Open Access is split into two payments: an Editorial Assessment Charge $(€ 2,190)$, which is payable after the Guided Open Access suitability check is passed and also covers the Editor Assessment Report, and the remainder of the APC, which is payable after acceptance in one of the three journals (a top-up fee of $€ 2,600$ for Nature Genetics or Nature Communications or $€ 800$ for Communications Biology). The total fee for Guided Open Access publication in Nature Genetics is $€ 4,790$, approximately half the regular Open Access APC.

The Editorial Assessment Report provides useful information and guidance, and is designed to help authors navigate the publication process more easily. This report includes an editorial summary and evaluation of the work; specific feedback on how to increase the reproducibility of the research to align with open research practices; editorial guidance on interpreting the reviewer comments; and clear advice on the next steps for further consideration of technically sound research. If publication is declined, authors can make the reviewer reports portable to other journals from other publishers.

Why might Guided Open Access be of interest as a publication option for some Nature Genetics authors? First, authors can be simultaneously considered at Nature Genetics, Nature Communications and Communications Biology, without a need for resubmission or transfers. Second, editors work collaboratively to guide authors throughout the process to help manuscripts find their best home, giving detailed evaluation and recommendation in the Editor Assessment Report. Finally, Guided Open Access allows for Open Access publication in Nature Genetics at a lower APC. We note that we follow the same editorial standards for all submissions regardless of which option is chosen.

We also believe that Guided Open Access could be appealing for consortium packages of coordinated papers that aim is to have a collection of papers published across journals, with coordinated publication times. Authors can prepare their submissions and submit just once. The manuscript will be assessed by editors across different journals. The peer-review process will be handled in the same cross-journal manuscript-tracking system, thus enhancing the ability to coordinate. Editors from multiple journals will discuss the manuscript and the reviews and make detailed, tailored recommendations for revisions. There is no need for transfers, because the entire process happens in one place, and all editors are equally informed of the progress in real time. In addition, even though authors will receive guidance from a committee of editors across journals, they will still have a champion editor who will handle the manuscript and serve as a point of contact during the process.

If you are interested in trying Guided Open Access or if you have any questions, please get in touch with us. We are happy to answer any questions.

Published online: 8 April 2021

https://doi.org/10.1038/s41588-021-00853-8 